\title{
THE BEHAVIOR OF FOURIER TRANSFORMS FOR NILPOTENT LIE GROUPS
}

\author{
RONALD L. LIPSMAN AND JONATHAN ROSENBERG
}

\begin{abstract}
We study weak analogues of the Paley-Wiener Theorem for both the scalar-valued and the operator-valued Fourier transforms on a nilpotent Lie group $G$. Such theorems should assert that the appropriate Fourier transform of a function or distribution of compact support on $G$ extends to be "holomorphic" on an appropriate complexification of (a part of) $\hat{G}$. We prove the weak scalar-valued Paley-Wiener Theorem for some nilpotent Lie groups but show that it is false in general. We also prove a weak operator-valued Paley-Wiener Theorem for arbitrary nilpotent Lie groups, which in turn establishes the truth of a conjecture of Moss. Finally, we prove a conjecture about Dixmier-Douady invariants of continuous-trace subquotients of $C^{*}(G)$ when $G$ is two-step nilpotent.
\end{abstract}

\section{INTRODUCTION}

The classical Paley-Wiener Theorems characterize the Fourier transforms of various classes of generalized functions of compact support on $\mathbb{R}^{n}$, as classes of holomorphic functions on $\mathbb{C}^{n}$ with exponential growth in imaginary directions and satisfying suitable growth or decay conditions in real directions, depending on the class of functions or distributions considered. A long-standing problem in abstract harmonic analysis has been to find analogues of these theorems with $\mathbb{R}^{n}$ replaced by a non-commutative locally compact group. In searching for such analogues, one immediately encounters four difficulties:

(0.1) It is not clear what the correct analogue of the Fourier transform should be for a (generalized) function $\varphi$ on a non-commutative locally compact group $G$. In general there are two main candidates, the operator-valued Fourier transform $\hat{\varphi}_{\mathrm{op}}: \pi \mapsto \pi(\varphi)$, with $\pi$ ranging over $\hat{G}$, the set of equivalence classes of irreducible unitary representations of $G$, and (when it makes sense) the scalar-valued Fourier transform $\hat{\varphi}: \pi \mapsto \operatorname{Tr} \pi(\varphi)$. However, depending on the context, it might be appropriate to consider non-unitary representations as well. (Still another candidate for a Fourier transform, based more on the theory of deformation quantization than on representation theory, is discussed in $[$ ArnG]. We will not consider it in this paper.)

(0.2) Since different representations of $G$ act on different Hilbert (or Banach or Fréchet) spaces, and representations are only defined up to equivalence, it is not clear how to study the variation of $\pi(\varphi)$ with $\pi$.

Received by the editors September 4, 1994.

1991 Mathematics Subject Classification. Primary 22E27; Secondary 43A30, 44A12, 22D25.

Key words and phrases. Nilpotent Lie group, scalar-valued Fourier transform, operator-valued Fourier transform, Paley-Wiener Theorem, Radon transform, Dixmier-Douady class.

Both authors were partially supported by NSF grant DMS-92-25063. 
(0.3) In general, a "dual" object for a non-commutative group, for example $\hat{G}$, has "singularities," due to the fact that representations tend to come in "series" which can degenerate or run into one another. It is not clear what sort of continuity, let alone holomorphicity, to expect of Fourier transforms at these singularities.

(0.4) When $G=\mathbb{R}^{n}, \hat{G} \cong \mathbb{R}^{n}$ has a natural complexification, $\mathbb{C}^{n}$. For a general locally compact group $G$, even leaving aside the problem of singularities, it is not clear how to define an analytic structure on $\hat{G}$.

Our objective in this paper is to discuss some methods for overcoming these difficulties to obtain information about the Fourier transform in the case of nilpotent Lie groups. In fact, connected and simply connected nilpotent Lie groups are the non-commutative groups "closest in appearance" to $\mathbb{R}^{n}$. Although we shall make some progress in studying Fourier analysis on them, we are still very far from a complete understanding.

Let $G$ be a connected and simply connected nilpotent Lie group with Lie algebra $\mathfrak{g}$. The Kirillov orbit method identifies $\hat{G}$ with the quotient space $\mathfrak{g}^{*} / G$ of the dual of $\mathfrak{g}$ under the coadjoint action, even as a topological space [Br, Joy]. We denote the irreducible representation attached to an orbit $\mathcal{O}$ (well-defined up to unitary equivalence) by $\pi_{\mathcal{O}}$, or by $\pi_{f}$ if $\mathcal{O}=G \cdot f$. For $\varphi \in C_{c}^{\infty}(G)$ and $\pi \in \hat{G}, \pi(\varphi)$ is a trace-class operator; furthermore, $\varphi \mapsto \operatorname{Tr} \pi(\varphi)$ is a tempered distribution on $G$ (though not necessarily a measure, as it is for semisimple Lie groups). Thus for $\varphi \in C_{c}^{\infty}(G)$, the scalar-valued Fourier transform of $\varphi$ is well-defined as a map $\hat{\varphi}$ : $\mathfrak{g}^{*} / G \rightarrow \mathbb{C}, \quad \mathcal{O} \mapsto \operatorname{Tr} \pi_{\mathcal{O}}(\varphi)$. When $G$ is nonabelian, the linear map $\varphi \mapsto \hat{\varphi}$ is never injective, but one can hope to characterize its image as a space of functions on $\mathfrak{g}^{*} / G$ satisfying certain continuity and holomorphicity conditions. Such a characterization we will call a strong scalar-valued Paley-Wiener Theorem for G. As far as we know, there is almost nothing in the literature on such theorems, except in very special cases.

The operator-valued Fourier transform $\hat{\varphi}_{\mathrm{op}}: \varphi \mapsto \pi(\varphi)$ for a nilpotent Lie group is harder to define as an actual operator-valued function, for the reason discussed in (0.2) above. Nevertheless, the following comments are in order:

(0.5) If $\varphi \in L^{1}(G), \hat{\varphi}_{\text {op }}$ makes sense as an element of the $C^{*}$-algebra $C^{*}(G)$ (the universal $C^{*}$-algebra completion of $L^{1}(G)$ ). Via the theory of realization of $C^{*}$-algebras as algebras of sections of bundles (see e.g. [Fell] or [Ros]), $\hat{\varphi}_{\text {op }}$ can then often be interpreted as a section of a bundle whose fibers are $C^{*}$-algebras. If (and only if) this bundle can be trivialized, then the operator-valued Fourier transform of $\varphi$ can be interpreted as an ordinary operator-valued function. Even when this is the case, the precise form of the function will depend on the trivialization of the bundle.

(0.6) If $\varphi$ is a tempered distribution on $G$ (and not necessarily an $L^{1}$ function), then for $\pi \in \hat{G}, \pi(\varphi)$ makes sense as an unbounded closed operator on the Hilbert space of $\pi$, or else as a continuous operator on the Fréchet space of $C^{\infty}$-vectors of the the representation. Both interpretations give the same (unambiguous) notion of vanishing of $\hat{\varphi}_{\text {op }}$ at a specific representation $\pi$.

(0.7) Since the map $\varphi \mapsto \hat{\varphi}_{\text {op }}$ from $L^{1}(G)$ to $C^{*}(G)$ is injective, one can hope to describe the operator-valued Fourier transform as a specific bijection from certain spaces of functions or distributions on $G$ to certain subspaces of $C^{*}(G)$ or spaces of operator-valued functions. Such a description we will call a 
strong operator-valued Paley-Wiener Theorem for G. As far as we know, such theorems have been proved only for Heisenberg groups and certain other special cases: see [Ando], [Kum], and [Sev1, Sev2].

There seems to be very little hope for proving either scalar-valued or operatorvalued strong Paley-Wiener Theorems for arbitrary nilpotent Lie groups. However, limited progress has been made in proving weaker substitutes. The papers [Moss] and [Park], motivated in part by [ScSi], proposed and managed to prove certain cases of:

0.8. Moss's Conjecture. Let $G$ be a connected and simply connected nilpotent Lie group, and let $\varphi$ be a distribution on $G$ with compact support. If $\hat{\varphi}_{\mathrm{op}}$ vanishes on a set of positive Plancherel measure in $\hat{G}$, then $\varphi=0$.

One of the goals of this paper is to prove this conjecture in the generality in which we have stated it. (Moss and Park only studied the case where $\varphi$ is given by an $L^{\infty}$ function, and used methods which do not generalize to arbitrary nilpotent Lie groups.) We will in fact prove in $\S 2$ something much more precise (see Corollary 2.15 below), which one can call a weak operator-valued Paley-Wiener Theorem for $G$. Here we rely on fundamental work of Niels Pedersen [Ped1, Ped2], which asserts that one can partition $\mathfrak{g}^{*} / G$ into a finite union of algebraic varieties defined over $\mathbb{R}$, in such a way that the representations corresponding to the Kirillov orbits in any given set of the partition can all be realized on a fixed Hilbert space in a very convenient way. Once one does this, our weak operator-valued Paley-Wiener Theorem asserts that for $\varphi$ a distribution with compact support, $\pi \mapsto \hat{\varphi}_{\mathrm{op}}(\pi)$ is "holomorphic" over each variety in the partition, in the sense that a large collection of its matrix coefficients extend to be analytic functions on the complexification of that variety.

Our search for a proof of Moss's conjecture led us also to try to prove:

0.9. Conjectured weak scalar-valued Paley-Wiener Theorem. For $\varphi \in$ $C_{c}^{\infty}(G)$, the scalar-valued Fourier transform $\hat{\varphi}$ extends to be holomorphic on a covering of the complexification of some algebraic variety defined over $\mathbb{R}$ which parameterizes the "generic" irreducible unitary representations of $G$.

Remark. A quick calculation for the Heisenberg group of dimension $2 n+1$ shows that this couldn't be true without passage to a covering, since the formula for $\hat{\varphi}$ involves a factor of $|\xi|^{-n}$, where $\xi$ is the parameter giving the central character of the representation. When $n$ is odd, this factor would appear not to be holomorphic in $\xi$, but we can rewrite it as $\left(\xi^{2 n}\right)^{-\frac{1}{2}}$, which now extends to a holomorphic function on the double cover of the punctured $\xi$-plane.

In $\S 1$ of this paper, we prove this weak scalar-valued Paley-Wiener Theorem for certain nilpotent Lie groups, and show how it leads to a proof of Moss's conjecture for these groups. More surprisingly, however, we show that Conjecture 0.9 is false in general. The analogue of Moss's conjecture also fails for the scalar-valued Fourier transform: it is possible for $\hat{\varphi}$ to vanish on a set of positive Plancherel measure in $\hat{G}$ without vanishing identically. Curiously, this fact is related to the Paley-Wiener Theorem for the classical Radon transform, as discussed in [Hel]. The analysis of certain counterexamples, which is also in $\S 1$, shows however that there is a sense in which Conjecture 0.9 is "close enough" to being true so that one can still deduce proofs of some cases of Moss's conjecture from an analysis of the scalar-valued Fourier transform. 
Finally, in $\S 3$ of this paper, we study the problem raised in (0.5) above about trivialization of the bundles of $C^{*}$-algebras which are implicit in the operator-valued Fourier transform. For 2-step nilpotent groups, we are able to prove a conjecture about this formulated by one of us in [RaRo, §4] and in [Ros, §3], thereby extending results in [Echt, $\S 6]$.

\section{The SCAlar-VAlued Fourier transform}

In this section we let $G$ denote a connected and simply connected nilpotent Lie group with Lie algebra $\mathfrak{g}$, and we study the scalar-valued Fourier transform $\varphi \mapsto \hat{\varphi}$. Note that $\hat{\varphi}$ is well-defined as a function on $\hat{G}$ if $\varphi \in C_{c}^{\infty}(G)$, but that also (by the Plancherel Theorem) $\hat{\varphi}(\pi)$ is well-defined for almost all $\pi$ (with respect to Plancherel measure) if $\varphi=\varphi_{1} * \varphi_{2}, \varphi_{1}, \varphi_{2} \in L^{1}(G) \cap L^{2}(G)$. In this latter case, the exceptional null set of $\pi$ 's where $\hat{\varphi}(\pi)$ is undefined will depend on $\varphi$.

A weak scalar-valued Paley-Wiener Theorem and Moss's conjecture for nilpotent Lie groups with square-integrable representations. Conjecture 0.9 , the weak scalar-valued Paley-Wiener Theorem, would if true be a natural generalization of the fact that the Fourier transform of $\varphi \in C_{c}^{\infty}\left(\mathbb{R}^{n}\right)$ extends to an entire holomorphic function on $\mathbb{C}^{n}$. In this subsection we will observe that a slightly more precise form of Conjecture 0.9 holds for nilpotent Lie groups with square-integrable representations, and leads immediately to an easy proof of Moss's Conjecture (0.8) for these groups. Then in the next subsection we will carry out a similar analysis for a much larger class of nilpotent Lie groups, and will find that Conjecture 0.9 fails even for some two-step nilpotent groups.

First we recall some of the theory developed in [MoorWo], for which an alternative reference is [CorGr, $\S 4.5]$. Let $G$ be a connected and simply connected nilpotent Lie group. If $\pi$ is an irreducible unitary representation of $G$, the projective kernel of $\pi$ is the set of elements of $G$ mapped under $\pi$ to scalar operators. This is always a connected normal subgroup $N$ of $G$, containing the center $Z$ of $G$. The representation $\pi$ is said to be square-integrable modulo $N$ if the absolute values of its matrix coefficients descend to $L^{2}$ functions on $G / N$. By [MoorWo, Theorem 1], this is equivalent to the condition that the Kirillov orbit corresponding to $\pi$ be flat, i.e., of the form $\mathcal{O}=f+\mathfrak{n}^{\perp}$, where $\mathfrak{n}$ is the Lie algebra of $N$, and also equivalent to the condition that the alternating bilinear form $B_{f}$, defined by $B_{f}(X, Y)=f([X, Y])$, should descend to a non-degenerate form on $\mathfrak{g} / \mathfrak{n}$.

1.1. Theorem. Let $G$ be a connected and simply connected nilpotent Lie group with Lie algebra $\mathfrak{g}$. Let $Z$ and $\mathfrak{z}$ be the centers of $G$ and $\mathfrak{g}$, respectively, and suppose $G$ has irreducible unitary representations which are square-integrable modulo $Z$. Fix Lebesgue measures on $\mathfrak{z}$ and on $\mathfrak{g} / \mathfrak{z}$, and take Lebesgue measure on $\mathfrak{g}$ to be the product of these two. Relative to our fixed Lebesgue measure on $\mathfrak{g} / \mathfrak{z}$, for $f \in \mathfrak{g}^{*}$, we can define $P(f)$, the Pfaffian polynomial of the alternating bilinear form $B_{f}$, viewed as an alternating form on $\mathfrak{g} / \mathfrak{z}$. (As a function of $f \in \mathfrak{g}^{*}$, it only depends on the image $\dot{f}$ of $f$ in $\mathfrak{g}^{*} / \mathfrak{z}^{\perp}=\mathfrak{z}^{*}$.) Let $V(\mathbb{R})=\left\{\dot{f} \in \mathfrak{z}^{*}: P(f) \neq 0\right\}$, and let $V(\mathbb{C})$ be its complexification $\left\{\dot{f} \in \mathfrak{z}_{\mathbb{C}}^{*}: P(f) \neq 0\right\}$. Then $V$ is a smooth affine algebraic variety defined over $\mathbb{R}$, connected in the (complex) Zariski topology and with $V(\mathbb{R})$ Zariski-dense in $V(\mathbb{C})$, and there is a natural map $\psi: V(\mathbb{R}) \rightarrow \mathfrak{g}^{*} / G$ having the following properties:

(a) $\psi$ is injective, and the complement in $\mathfrak{g}^{*}$ of the inverse image of $\psi(V(\mathbb{R}))$ has Lebesgue measure zero. 
(b) The pull-back under $\psi$ of some quasi-image in $\mathfrak{g}^{*} / G$ of Lebesgue measure on $\mathfrak{g}^{*}$ is quasi-equivalent to a smooth measure on $V(\mathbb{R})$.

(c) For $\varphi \in C_{c}^{\infty}(G), v \mapsto \hat{\varphi}(\psi(v))$ extends to a holomorphic function on a double cover of $V(\mathbb{C})$.

Furthermore, if $\varphi \in C_{c}^{\infty}(G)$ and if $\hat{\varphi}_{\mathrm{op}}$ vanishes on a set of positive Plancherel measure in $\hat{G}$, it follows that $\varphi \equiv 0$.

Proof. (i) Define $\psi$ by sending $\dot{f} \in V(\mathbb{R})$ to $f+\mathfrak{z}^{\perp}$, where $f$ lies in the inverse image of $\dot{f}$ in $\mathfrak{g}^{*}$. By [MoorWo], this gives a bijection from $V(\mathbb{R})$ to the set of $G$-orbits in $\mathfrak{g}^{*}$ corresponding to irreducible unitary representations which are square-integrable modulo $Z$. The inverse image of $\psi(V(\mathbb{R}))$ in $\mathfrak{g}^{*}$ is $\left\{f \in \mathfrak{g}^{*}: P(f) \neq 0\right\}$, which is obviously a Zariski-open subset of $\mathfrak{g}^{*}$, and is non-empty by the hypothesis that $B_{f}$ is non-degenerate on $\mathfrak{g} / \mathfrak{z}$ for at least one $f \in \mathfrak{g}^{*}$. Properties (a) and (b) are obvious. Since $V$ is a non-empty Zariski-open subset of an affine space, defined by the non-vanishing of the polynomial $P$ which has real coefficients, it is a smooth affine algebraic variety defined over $\mathbb{R}$, connected in the (complex) Zariski topology and with $V(\mathbb{R})$ Zariski-dense in $V(\mathbb{C})$. Furthermore, since $V$ is Zariski-connected, $V(\mathbb{C}$ ) is connected in the usual Hausdorff topology (for a proof, see [Mum, Corollary 4.16] or [Shaf, Ch. VII, $\S 2]$ ). (Here it is essential to take complex points; $V(\mathbb{R})$ may not be connected in the usual Hausdorff topology.) By [MoorWo, Theorem 6], the Plancherel measure on $\hat{G}$ corresponds under $\psi$ to a constant multiple of the smooth measure $|P(\dot{f})| d \dot{f}$ on $V(\mathbb{R})$. (The constant only depends on the dimensions of $\mathfrak{g}$ and $\mathfrak{z}$.)

(ii) Now let $\varphi \in C_{c}^{\infty}(G)$. We compute the distribution character of $\pi_{\psi(\dot{f})}$ on $\varphi$ using the inversion theorem for the abelian Fourier transform as in [MoorWo, pp. 458-459], and find that

$$
\hat{\varphi}(\psi(\dot{f}))=\operatorname{Tr} \pi_{\psi(\dot{f})}(\varphi)=\frac{c}{|P(f)|} \tilde{\varphi}(\dot{f}),
$$

where $\tilde{\varphi}$ is the (abelian) Fourier transform of $\left.(\varphi \circ \exp )\right|_{\mathfrak{z}}$, and $c$ is a constant depending only on our normalizations of Lebesgue measures on $\mathfrak{g}$ and $\mathfrak{z}$. Clearly $\tilde{\varphi}(\dot{f})$ belongs to the Paley-Wiener class $\mathcal{P} \mathcal{W}\left(\mathfrak{z}^{*}\right)$, and so $|P(f)| \hat{\varphi} \circ \psi(\dot{f})$ extends to be entire holomorphic on $\mathfrak{z}_{\mathbb{C}}^{*}$. Furthermore, the extra factor $|P(f)|$ may be rewritten as $\left(P(f)^{2}\right)^{\frac{1}{2}}$, which makes sense as a holomorphic function on the double cover of $V(\mathbb{C})$, so (c) is satisfied. Finally, if $\hat{\varphi} \circ \psi$ vanishes on a set of positive smooth measure in $V(\mathbb{R})$, we deduce that its analytic continuation on the double cover of $V(\mathbb{C})$ vanishes on a set of positive smooth measure, and thus must vanish everywhere since the double cover of $V(\mathbb{C})$ is connected.

(iii) Now suppose $\hat{\varphi}_{\mathrm{op}}(\pi)=\pi(\varphi)$ vanishes for $\pi$ in a set $E$ of positive Plancherel measure in $\hat{G}$. Let $\lambda$ be the left regular representation of $G$. Then for any $g \in G$, $\pi(\lambda(g) \varphi)=\pi(g) \pi(\varphi)$ also vanishes for $\pi \in E$, and in particular its trace is zero. So $\widehat{\lambda(g) \varphi}$ vanishes on $E$, and by (ii) above, $\widehat{\lambda(g) \varphi} \circ \psi \equiv 0$. Now by the Plancherel Theorem, we have

$$
\varphi\left(g^{-1}\right)=(\lambda(g) \varphi)(1)=\mathrm{const} \int_{V(\mathbb{R})} \widehat{\lambda(g) \varphi} \circ \psi(\dot{f})|P(\dot{f})| d \dot{f}=0
$$

for all $g$, i.e., $\varphi \equiv 0$. 
1.3. Corollary. If $G$ has square-integrable representations modulo $Z$, then Moss's Conjecture (0.8) holds for arbitrary distributions $\varphi$ of compact support on $G$.

Proof. It is sufficient to observe that Moss's Conjecture for functions in $C_{c}^{\infty}(G)$ implies the same conjecture for arbitrary distributions $\varphi$ of compact support on $G$. Suppose $\varphi$ is such a distribution and $\hat{\varphi}_{\mathrm{op}}$ vanishes on a set $E$ of positive Plancherel measure. Then for any function $\theta \in C_{c}^{\infty}(G), \widehat{\varphi * \theta}_{\text {op }}=\hat{\varphi}_{\text {op }} \cdot \hat{\theta}_{\text {op }}$ also vanishes on $E$. Since the convolution $\varphi * \theta$ is in $C_{c}^{\infty}(G)$, we deduce from Theorem 1.1 that $\varphi * \theta=0$. This being true for all $\theta, \varphi=0$ as a distribution.

Failure of the weak scalar-valued Paley-Wiener Theorem, but a proof of Moss's conjecture, for groups whose generic orbits are flat. We proceed now to examine what happens to the proof of Theorem 1.1 when $G$ is a connected, simply connected nilpotent Lie group whose generic orbits are flat, i.e., whose generic irreducible unitary representations are square-integrable modulo their projective kernels, but for which the projective kernel varies depending on the representation. As we shall see, the class of groups with this property includes all two-step nilpotent Lie groups, and some higher-step groups as well.

1.4. Theorem. Let $G$ be a connected and simply connected nilpotent Lie group with Lie algebra $\mathfrak{g}$, and let $2 d$ be the maximal dimension of $G$-orbits in $\mathfrak{g}^{*}$. Assume that there is a non-empty $G$-invariant Zariski-open subset $\Omega$ of $\mathfrak{g}^{*}$ consisting of flat orbits of dimension $2 d$. Then (after perhaps modifying $\Omega$, keeping the same conditions), there is a smooth quasi-projective variety $W(\mathbb{R})$ parametrizing a set of abelian ideals $\mathfrak{n} \triangleleft \mathfrak{g}$ of codimension $2 d$, such that $\Omega / G$ can be identified with a Zariski-open subset $V(\mathbb{R})$ of

$$
E(\mathbb{R})=\left\{(\mathfrak{n}, f): \mathfrak{n} \in W(\mathbb{R}), f \in \mathfrak{n}^{*}, f \perp[\mathfrak{g}, \mathfrak{n}]\right\} .
$$

Here $V(\mathbb{R})=\Omega / G$ and $E(\mathbb{R})$ are each the set of real points of a quasi-projective algebraic variety defined over $\mathbb{R}$, and $\Phi: E(\mathbb{R}) \rightarrow W(\mathbb{R})$ sending $(\mathfrak{n}, f)$ to $\mathfrak{n}$ is an algebraic vector bundle. The Plancherel measure class on $\hat{G}$ corresponds to the smooth measure class on $V(\mathbb{R})$. For $\varphi \in C_{c}^{\infty}(G)$, the restriction of $\hat{\varphi}$ to any fiber of $\Phi$ extends to be holomorphic on a double cover of the complexification of that fiber. If $\hat{\varphi}_{\mathrm{op}}$ vanishes on a set of positive Plancherel measure in $\hat{G}$, then $\varphi=0$. However, it is possible for $\hat{\varphi}$ to be non-zero on a set of positive Plancherel measure and yet to vanish identically on another set of positive Plancherel measure.

Proof. Let $U(\mathbb{R})$ be the Grassmann manifold of $2 d$-dimensional linear subspaces of $\mathfrak{g}^{*}$, which by duality we can identify with the Grassmann manifold of $2 d$-codimensional linear subspaces of $\mathfrak{g}$. As is well known, this can be identified with the real points of a smooth projective variety $U$. The "Gauss map" $\Phi: \Omega \rightarrow U(\mathbb{R})$ sending $f \in \Omega$ to the tangent space at $f$ to the variety $G \cdot f$ is a morphism of smooth varieties (since all the orbits in $\Omega$ have the same dimension $2 d$ ), and $\Phi$ is constant on $G$-orbits by the assumption that the orbits in $\Omega$ are flat. The image of $\Phi$ is contained in the set $Y$ of annihilators of abelian ideals ${ }^{1} \mathfrak{n}$ in $\mathfrak{g}$ of codimension $2 d$, and this is a closed algebraic subset of $U(\mathbb{R})$ since being an abelian ideal is equivalent to a finite number of (real) polynomial conditions. Cutting $\Omega$ down if

\footnotetext{
${ }^{1}$ They are ideals since the orbits are flat [CorGr, Theorem 3.2.3], and are abelian by the condition that the orbits have maximal dimension [Dix2, Proposition 1.11.7].
} 
necessary to a Zariski-open subset (see for example [Shaf, Ch. I, §5, Theorem 6]), we may suppose the image of $\Phi$ is a smooth variety $W(\mathbb{R})$, which will consist of annihilators of abelian ideals $\mathfrak{n}$ in $\mathfrak{g}$ of codimension $2 d$ which are also of the form $\mathfrak{g}_{f}$ for some $f \in \mathfrak{g}^{*}$. For any such $\mathfrak{n}$, one has square-integrable representations modulo $\mathfrak{n}$ corresponding to all $f \in \mathfrak{n}^{*} \cap[\mathfrak{g}, \mathfrak{n}]^{\perp}$ for which the Pfaffian $P_{\mathfrak{n}}(f)$ of the induced form on $\mathfrak{g} / \mathfrak{n}$ is non-zero. For any such pair $(\mathfrak{n}, f)$, let $\varphi \in C_{c}^{\infty}(G)$ and $\mathcal{O}=f+\mathfrak{n}^{\perp}$. We compute the distribution character of $\pi_{\mathcal{O}}$ on $\varphi$ using the inversion theorem for the abelian Fourier transform as in [MoorWo, pp. 458-459], and find that

$$
\hat{\varphi}(\mathcal{O})=\operatorname{Tr} \pi_{\mathcal{O}}(\varphi)=\frac{c}{\left|P_{\mathfrak{n}}(f)\right|} \tilde{\varphi}(f),
$$

where $\tilde{\varphi}$ is the (abelian) Fourier transform of $\left.(\varphi \circ \exp )\right|_{\mathfrak{n}}$, and $c$ is a constant depending only on the normalizations of Lebesgue measures on $\mathfrak{g}$ and $\mathfrak{n}$. (Note here that for fixed $\mathcal{O}=f+\mathfrak{n}^{\perp}, f$ is only defined up to an element of $\mathfrak{n}^{\perp}$, but gives a well-defined class in $\mathfrak{g}^{*} / \mathfrak{n}^{\perp}=\mathfrak{n}^{*}$, so that $\tilde{\varphi}(f)$ is well-defined. Similarly, $P_{\mathfrak{n}}(f)$ only depends on the image of $f$ in $\mathfrak{n}^{*}$.) Since the polynomial $P_{\mathfrak{n}}$ is not identically zero, there is a non-empty Zariski-open subset of $(\mathfrak{n} /[\mathfrak{g}, \mathfrak{n}])^{*}$ on which $P_{\mathfrak{n}}(f) \neq 0$, and the character formula $(1.5)$ is valid for all such $f$. Clearly $\tilde{\varphi}(f)$ belongs to the PaleyWiener class $\mathcal{P} \mathcal{W}\left(\mathfrak{n}^{*}\right)$, and so $\left|P_{\mathfrak{n}}(f)\right| \hat{\varphi}(G \cdot f)$ extends to be entire holomorphic on $\mathfrak{n}_{\mathbb{C}}^{*}$. By general Kirillov theory, Plancherel measure on $\hat{G}$ must correspond to a smooth measure on $V(\mathbb{R})$. From the character formula (1.5), if $\varphi \circ \exp$ vanishes identically on a set of $\mathfrak{n}$ 's of positive measure in $W(\mathbb{R})$, then $\hat{\varphi}$ will vanish on a set of positive Plancherel measure. This can happen without $\varphi$ vanishing identically. (A specific counterexample will be given in Example 1.8 below.)

Now let us verify Moss's conjecture for $G$. By the proof of Corollary 1.3, it is enough to verify the conjecture for $\varphi \in C_{c}^{\infty}(G)$. Assume that for such a $\varphi, \hat{\varphi}_{\text {op }}$ vanishes on a set $E$ of positive Plancherel measure. By Fubini's Theorem, there is a set $E_{1}$ of positive smooth measure in $W(\mathbb{R})$ such that for each $\mathfrak{n} \in E_{1}, \hat{\varphi}_{\text {op }}$ vanishes on a set of $\pi_{\mathcal{O}}$ 's with $\mathcal{O}=f+\mathfrak{n}^{\perp}$ and with $f$ ranging over a set of positive Lebesgue measure in $(\mathfrak{n} /[\mathfrak{g}, \mathfrak{n}])^{*}$. Then for any $g \in G$ and $\mathfrak{n} \in E_{1}, \widehat{\lambda(g) \varphi}$ vanishes at $f+\mathfrak{n}^{\perp}$ for $f$ ranging over a set of positive Lebesgue measure in $(\mathfrak{n} /[\mathfrak{g}, \mathfrak{n}])^{*}$. Since $\tilde{\varphi}(f)$ is holomorphic on $\mathfrak{n}_{\mathbb{C}}^{*},(1.5)$ implies that the abelian Fourier transform of $\left.(\lambda(g) \varphi) \circ \exp \right|_{\mathfrak{n}}$ vanishes identically on $(\mathfrak{n} /[\mathfrak{g}, \mathfrak{n}])^{*}$ for all $g \in G$ and all $\mathfrak{n} \in E_{1}$, and thus for such $\mathfrak{n}$ and any $g$, the integral of $(\lambda(g) \varphi) \circ$ exp over any coset in $\mathfrak{n}$ of $[\mathfrak{g}, \mathfrak{n}]$ vanishes by Fourier inversion. Since

$$
\int_{[\mathfrak{g}, \mathfrak{n}]}(\lambda(\exp -Y) \varphi) \circ \exp (X) d X=\int_{[\mathfrak{g}, \mathfrak{n}]} \varphi \circ \exp (Y+X) d X,
$$

(the Jacobian of the change of variables that comes in is 1 since $\mathfrak{g}$ is nilpotent), the integral of $\varphi \circ$ exp over any coset in $\mathfrak{g}$ of $[\mathfrak{g}, \mathfrak{n}]$ vanishes. Let $r=\operatorname{dim}[\mathfrak{g}, \mathfrak{n}]=$ $\operatorname{dim} W(\mathbb{R})$, and consider the $r$-dimensional Radon transform

$$
\widehat{\varphi \circ \exp }_{\text {Radon }}(\sigma, X)=\int_{\sigma} \varphi \circ \exp (Y+X) d Y
$$

where $\sigma$ ranges over $r$-dimensional subspaces of $\mathfrak{g}$. We have shown that

$$
\widehat{\varphi \exp }_{\text {Radon }}(\sigma, X)
$$


vanishes identically for $\sigma=[\mathfrak{g}, \mathfrak{n}], \mathfrak{n} \in E_{1}$. For $f \in \mathfrak{g}^{*}, k \in \mathbb{N}$, define the polynomial

$$
P_{k}(f)=\int_{\mathfrak{g}} \varphi \circ \exp (X) f(X)^{k} d X
$$

If this vanishes for all $k$, then $\varphi \equiv 0$ (using Stone-Weierstrass, for example). By the Paley-Wiener Theorem for the $r$-dimensional Radon transform [Hel, Ch. I, Corollary 6.4], what we know is that the restriction of $P_{k}$ to $[\mathfrak{g}, \mathfrak{n}]^{\perp}$ vanishes for each $\mathfrak{n} \in E_{1}$. This is enough to imply that each $P_{k}$ vanishes and thus that $\varphi$ vanishes.

1.6. Proposition. The hypotheses of Theorem 1.4 are satisfied if $G$ is a two-step connected and simply connected nilpotent Lie group. In fact, for such a group, any coadjoint orbit is flat.

Proof. Let $\mathfrak{g}$ be a two-step nilpotent Lie algebra with center $\mathfrak{z}$, and let the corresponding simply connected nilpotent Lie groups be $G$ and $Z$. The two-step assumption means $[\mathfrak{g}, \mathfrak{g}] \subseteq \mathfrak{z}$. If $f \in \mathfrak{g}^{*}$ and $X \in \mathfrak{g}$, then

$$
\begin{aligned}
(\exp (t X) \cdot f)(Y) & =f\left(e^{-t \operatorname{ad} X} Y\right) \\
& =f\left(Y-t[X, Y]+\frac{t^{2}}{2}[X,[X, Y]]+\cdots\right) \\
& =f(Y-t[X, Y])=(f-t f \circ \operatorname{ad} X)(Y),
\end{aligned}
$$

so $G \cdot f=f+\mathfrak{g}_{f}^{\perp}$ and the $G$-orbit through $f$ is flat, with $G_{f}$ as the projective kernel of the corresponding irreducible unitary representation. So the Zariski-open subset consisting of orbits of maximal dimension (see [Dix2, Proposition 1.11.5]) will satisfy the hypotheses of Theorem 1.4.

1.7. Remark. However, as observed in [MoorWo, p. 453], there is no upper bound on the nilpotent length of a nilpotent Lie group with generic representations squareintegrable modulo the center, hence a fortiori modulo their projective kernels. So Theorem 1.4 also applies to some higher-step groups.

1.8. Example. Here is a specific example of the situation in Theorem 1.4. Let $\mathfrak{g}$ be the free two-step nilpotent Lie algebra on 3 generators, with basis $X_{1}, \ldots, X_{6}$, where $\left[X_{6}, X_{5}\right]=X_{3},\left[X_{6}, X_{4}\right]=X_{1},\left[X_{5}, X_{4}\right]=X_{2}$, and $X_{1}, X_{2}, X_{3}$ are central. (In [Ped3], this example is labelled N6N15.) Let $G$ be the corresponding group. The center $\mathfrak{z}$ of $\mathfrak{g}$ is spanned by $X_{1}, X_{2}, X_{3}$. If $f \in \mathfrak{g}^{*}$ is "regular," then $\mathfrak{g}_{f}$ is an ideal containing $\mathfrak{z}$ with codimension 1 , and any such ideal arises in this way. Thus we see that $\mathfrak{g}_{\text {reg }}^{*} / G$ can be identified with a Zariski-open subset of the total space of a rank-two vector bundle over the set of possibilities for $\mathfrak{n}=\mathfrak{g}_{f}$, which in turn can be identified with the projective plane of one-dimensional subspaces of $\mathfrak{g} / \mathfrak{z}$. For any such $\mathfrak{n},[\mathfrak{g}, \mathfrak{n}]$ is a two-dimensional subspace of $\mathfrak{z}$, and $\mathfrak{n}$ and $[\mathfrak{g}, \mathfrak{n}]$ determine one another. In this case $P_{\mathfrak{n}}$ is a linear functional on $(\mathfrak{n} /[\mathfrak{g}, \mathfrak{n}])^{*}$. From (1.5), $\hat{\varphi}\left(f+\mathfrak{n}^{\perp}\right)\left|P_{\mathfrak{n}}(f)\right|$ is (up to a constant) the abelian Fourier transform of $\left.\varphi \circ \exp \right|_{\mathfrak{n}}$. Clearly one can choose $\varphi \neq 0 \in C_{c}^{\infty}(G)$ so that the support of $\varphi \circ$ exp does meet some possible $\mathfrak{n}$ but misses a set of $\mathfrak{n}$ 's of positive measure in the projective plane of one-dimensional subspaces of $\mathfrak{g} / \mathfrak{z}$. So vanishing of $\hat{\varphi}$ on a set of positive Plancherel measure does not imply vanishing of $\hat{\varphi}$ everywhere. 
In fact,

$$
\int_{(\mathfrak{n} /[\mathfrak{g}, \mathfrak{n}]) *} \hat{\varphi}\left(f+\mathfrak{n}^{\perp}\right)\left|P_{\mathfrak{n}}(f)\right| d \dot{f}=c \int_{[\mathfrak{g}, \mathfrak{n}]} \varphi \circ \exp .
$$

So we can compute in this way the integral of $\varphi \circ \exp$ over any two-dimensional subspace of $\mathfrak{z}$, in other words the Radon transform of $\left.\varphi \circ \exp \right|_{\mathfrak{z}}$, restricted only to those two-planes in $\mathfrak{z}$ which pass through the origin. Now we can relate the Plancherel formula for $G$ to the classical Radon inversion formula [Hel, Ch. I], which says that

$$
\varphi(1)=\frac{-1}{2 \pi} \int_{\substack{\omega \in \mathfrak{z} \\|\omega|=1}} \frac{d^{2}}{d p^{2}}\left\{\int_{\substack{z \cdot \omega \in \mathfrak{z} \\=}} \varphi \circ \exp \right\}_{p=0} d \omega .
$$

Rewriting this in terms of $\hat{\varphi}$ gives precisely the Plancherel formula for $G$. And a correct formulation of a weak Paley-Wiener Theorem for the scalar-valued Fourier transform on $G$ resembles the Paley-Wiener Theorem for the Radon transform in [Hel, Ch. I].

1.9. Remark. It should be clear from Theorem 1.4 and Example 1.8 that the weak Paley-Wiener Theorem for the scalar-valued Fourier transform can fail whenever the supports of the distribution characters of "generic" representations of $G$ "rotate around." In the examples we've examined here, the support of the distribution character has always been an ideal in $\mathfrak{g}$. While this is the case for low-dimensional nilpotent Lie groups, Pukanszky [Puk, p. 413] has given an interesting 7-dimensional example where the supports of the distribution characters of "generic" representations are quadric cones in $\mathfrak{g}$. The weak Paley-Wiener Theorem for the scalar-valued Fourier transform definitely fails in such cases as well.

\section{A Paley-Wiener Theorem for the OPERATOR-VALUED FOURIER TRANSFORM}

Pedersen's models for the irreducible unitary representations. In an important series of papers ([Ped1], [Ped2], which built on ideas of Pukanszky), Pedersen has given a detailed description of the fine structure of $\hat{G}$, for a nilpotent Lie group $G$, and he has elucidated very sharply the nature of the analytic objects that appear in that structure. In this section we utilize these objects to prove a weak version of the operator-valued Paley-Wiener Theorem.

The structure depends on a choice of a Jordan-Hölder basis for $\mathfrak{g}$ which we now fix: namely, a basis $X_{1}, X_{2}, \ldots, X_{m}$ for $\mathfrak{g}$ with the property that $\mathfrak{g}_{j}=$ $\operatorname{span}\left(X_{1}, \ldots, X_{j}\right)$ is an ideal in $\mathfrak{g}$ for each $1 \leq j \leq m$. Each $f \in \mathfrak{g}^{*}$ defines a bilinear form $B_{f}$ by $B_{f}(X, Y)=f([X, Y])$. The restriction of $B_{f}$ to each $\mathfrak{g}_{j}$ determines a set of jump indices

$$
J_{f}^{j}=\left\{1 \leq k \leq j: X_{k} \notin \mathfrak{g}_{k-1}+\operatorname{rad}\left(\left.B_{f}\right|_{\mathfrak{g}_{j}}\right)\right\},
$$

and $\mathfrak{g}^{*}$ is the finite union of the sets $\Omega_{\varepsilon}$ where the $m$-tuple of sets of jump indices $\left(J_{f}^{1}, \ldots, J_{f}^{m}\right)$ takes a fixed value $\varepsilon$. This is Pedersen's ("fine") stratification of $\mathfrak{g}^{*}$. Each $\Omega_{\varepsilon}$ is $G$-invariant. The non-empty sets $\Omega_{\varepsilon}$, of which there are only a finite number, may be numbered as $\Omega_{1}, \ldots, \Omega_{k}$ so that $\Omega_{1}$ is Zariski-open in $\mathfrak{g}^{*}$ and each successive $\Omega_{j}$ is a Zariski-open set in $\mathfrak{g}^{*} \backslash\left(\bigcup_{i<j} \Omega_{i}\right)$, hence is a real affine 
variety. Note that the last set in the stratification, $\Omega_{k}$, corresponds to the case where $\varepsilon=\emptyset$, and thus to the one-dimensional unitary representations of $G$. For any particular value of $\varepsilon$, all the orbits in $\Omega_{\varepsilon}$ have a fixed dimension $2 d$, which is also the cardinality of $J_{f}^{m}$, say $J_{f}^{m}=\left\{j_{1}<\cdots<j_{2 d}\right\}$. Then $X_{1}, X_{2}, \ldots, X_{m}$ can be viewed as linear coordinates $\xi_{1}, \xi_{2}, \ldots, \xi_{m}$ on $\mathfrak{g}^{*}$, and by [Ped2, Lemma 1.6.1], each $G$-orbit in $\Omega_{\varepsilon}$ contains a unique point where $\xi_{j_{1}}, \ldots, \xi_{j_{2 d}}$ each take the value 0 . Thus the intersection $\Lambda_{\varepsilon}$ of $\Omega_{\varepsilon}$ with the hyperplane

$$
\left\{\xi_{j_{1}}=0, \ldots, \xi_{j_{2 d}}=0\right\}
$$

is an affine variety parametrizing the $G$-orbits in $\Omega_{\varepsilon}$. The first basic result that we need is one of the main results of [Ped2]:

2.2. Theorem ([Ped2, Thm. 2.7.2]—see also [Ped2, Thm. 3.2.1]). Let $\mathcal{O}$ be an orbit in $\mathfrak{g}^{*}$ of dimension $2 d$. Then there exists a unique irreducible unitary representation $\pi$ of $G$ on $L^{2}\left(\mathbb{R}^{d}\right)$ such that $\mathcal{H}_{\pi}^{\infty}=\mathcal{S}\left(\mathbb{R}^{d}\right)$ and such that for every $X \in \mathfrak{g}$, there exist $d+1$ polynomial functions $a_{X, r}(0 \leq r \leq d)$, with real coefficients, in $d$ variables "associated to $\mathcal{O}$," which satisfy

$$
d \pi(X) f(t)=i a_{X, 0}(t) f(t)+\sum_{r=1}^{d} a_{X, r}(t) \frac{\partial f}{\partial t_{r}}(t)+\frac{1}{2} \sum_{r=1}^{d} \frac{\partial a_{X, r}}{\partial t_{r}}(t) f(t)
$$

for all $f \in \mathcal{S}\left(\mathbb{R}^{d}\right)$. The representation $\pi$ is in the equivalence class of irreducible unitary representations associated with the orbit $\mathcal{O}$.

What is derived in [Ped2], but not stated explicitly there, is the dependence of the polynomial functions $a_{X, r}$ on the affine parameters that determine $\mathcal{O}$ (or $\pi$ ). That computation constitutes the heart of $[\operatorname{Ped} 2, \S 5]$. We summarize the results of Pedersen's computation in a second theorem.

2.4. Theorem. Let $\Omega_{\varepsilon}$ be any stratum in Pedersen's stratification, and let $2 d$ be the dimension of orbits in this stratum. Parameterize the orbits in this stratum by the cross-section $\Lambda_{\varepsilon}$. Fix $X \in \mathfrak{g}$. Then the functions $a_{X, r}^{\lambda}$ of Theorem $2.1(0 \leq r \leq d)$, viewed as also depending on $\lambda \in \Lambda_{\varepsilon}$, are polynomials in $t$ with coefficients that are rational functions of $\lambda$. The denominators of these rational functions do not vanish on the complexification $\left(\Lambda_{\varepsilon}\right)_{\mathbb{C}}$ of $\Lambda_{\varepsilon}$.

Proof. See [Ped2, §5] and [Ped4, Remark 1.4.5]. It is shown in fact that the denominators of these rational functions may be expressed as products of certain explicit Pfaffians, nonvanishing on $\left(\Lambda_{\varepsilon}\right)_{\mathbb{C}}$.

Two representative examples. Now we give two examples taken from [Ped3], which will show how Theorems 2.2 and 2.4 can be applied to prove holomorphicity of the matrix coefficients in the affine variables that parameterize the orbits in a single stratum. While these examples are fairly straightforward, they illustrate virtually all of the features that show up in the general case.

2.5. Example. Let $\mathfrak{g}$ be the four-dimensional Lie algebra with Jordan-Hölder basis $X_{1}, X_{2}, X_{3}, X_{4}$, where $\left[X_{4}, X_{3}\right]=X_{2}$ and $\left[X_{4}, X_{2}\right]=X_{1}$, denoted by N4N1 in [Ped3]. Then $X_{1}, X_{2}, X_{3}, X_{4}$ can be considered as linear coordinates $\xi_{1}, \xi_{2}, \xi_{3}, \xi_{4}$ on $\mathfrak{g}^{*}$, and the open stratum $\Omega_{1}$ is given by $\xi_{1} \neq 0$. The corresponding jump indices 
are $j_{1}=2, j_{2}=4$. A cross-section for the orbits is given by $\Lambda_{1}$, the intersection of $\Omega_{1}$ with the hyperplane $\left\{\xi_{2}=\xi_{4}=0\right\}$. Thus coordinates on $\Lambda_{1}$ can be taken to be $\xi_{1} \neq 0$ and $\xi_{3}$. In this case $d=1$, and (2.3) becomes

$$
d \pi\left(X_{j}\right)= \begin{cases}i \xi_{1}, & j=1, \\ i t_{1}, & j=2, \\ \frac{1}{2 \xi_{1}}\left(2 i \xi_{1} \xi_{3}-i \xi_{2}^{2}+i t_{1}^{2}\right), & j=3, \\ \xi_{1} \frac{\partial}{\partial t_{1}}, & j=4,\end{cases}
$$

all acting on $\mathcal{S}(\mathbb{R})$. (Here $t_{1}$ is the coordinate on $\mathbb{R}$.)

Integrating, we see that

$$
\pi\left(\exp x_{j} X_{j}\right)= \begin{cases}e^{i x_{1} \xi_{1}}, & j=1, \\ e^{i x_{2} t_{1}}, & j=2, \\ \exp \left(\frac{i x_{3}}{2 \xi_{1}}\left(2 \xi_{1} \xi_{3}-\xi_{2}^{2}+t_{1}^{2}\right)\right), & j=3, \\ \text { translation by } x_{4} \xi_{1}, & j=4,\end{cases}
$$

all acting on $L^{2}(\mathbb{R})$.

Now let $f_{1}, f_{2} \in \mathcal{S}(\mathbb{R})$. We can compute the matrix coefficient $\left\langle\pi(g) f_{1}, f_{2}\right\rangle$ for fixed $g \in G$ as a function of $\xi_{1}$ and $\xi_{3}$, after setting $\xi_{2}=\xi_{4}=0$ :

$$
\begin{aligned}
\left\langle\pi\left(\exp x_{1} X_{1}\right)\right. & \left.\pi\left(\exp x_{2} X_{2}\right) \pi\left(\exp x_{3} X_{3}\right) \pi\left(\exp x_{4} X_{4}\right) f_{1}, f_{2}\right\rangle \\
& =\int_{\mathbb{R}} \exp \left(i\left(x_{1} \xi_{1}+x_{2} t+x_{3} \xi_{3}+\frac{x_{3} t^{2}}{2 \xi_{1}}\right)\right) f_{1}\left(t+x_{4} \xi_{1}\right) \overline{f_{2}(t)} d t \\
& =e^{i \xi_{1} x_{1}+i \xi_{3} x_{3}} \int_{\mathbb{R}} e^{i\left(x_{2} t+\frac{x_{3}}{2 \xi_{1}} t^{2}\right)} f_{1}\left(t+x_{4} \xi_{1}\right) \overline{f_{2}(t)} d t .
\end{aligned}
$$

The exponential factors and the factor involving $f_{1}$ are clearly everywhere holomorphic in $\xi_{1}$ and $\xi_{3}$ away from $\left\{\xi_{1}=0\right\}$, provided that $f_{1}$ has (ordinary) Fourier transform $\hat{f}_{1} \in C_{c}^{\infty}(\mathbb{R})$ and thus $f_{1}$ lies in the Paley-Wiener class $\mathcal{P} \mathcal{W}(\mathbb{R})$ (so that $f_{1}\left(t+x_{4} \xi_{1}\right)$ is entire in $\left.\xi_{1}\right)$. If $f_{2} \in C_{c}^{\infty}(\mathbb{R})$, there is no problem with convergence of the integral, so the matrix coefficient is holomorphic in $\left(\Lambda_{1}\right)_{\mathbb{C}}$.

Caution. Because of the factor $f_{1}\left(t+x_{4} \xi_{1}\right)$ in the integrand and the growth of the factor $\exp \left(i \frac{x_{3} t^{2}}{2 \xi_{1}}\right)$ in $t$ for imaginary $\xi_{1}$, the matrix coefficient will not be holomorphic in $\xi_{1}$ for arbitrary $f_{1}, f_{2} \in \mathcal{S}(\mathbb{R})$. Indeed, if $x_{1}=x_{2}=x_{3}=0$, the matrix coefficient reduces to

$$
\left\langle\pi\left(\exp x_{4} X_{4}\right) f_{1}, f_{2}\right\rangle=g\left(x_{4} \xi_{1}\right),
$$

where $g=f_{1} * f_{2}^{*}, f_{2}^{*}(t)=\overline{f_{2}(-t)}$. This is a Schwartz function but it may or may not be real analytic, let alone entire.

2.6. Example. Let $\mathfrak{g}$ be the five-dimensional Lie algebra with Jordan-Hölder basis $X_{1}, X_{2}, X_{3}, X_{4}, X_{5}$, where $\left[X_{5}, X_{4}\right]=X_{3},\left[X_{5}, X_{3}\right]=X_{2},\left[X_{5}, X_{2}\right]=X_{1}$ and $\left[X_{4}, X_{3}\right]=X_{1}$, denoted by $N 5 N 6$ in [Ped3]. Then, as in the preceding example, $X_{1}, X_{2}, X_{3}, X_{4}, X_{5}$ can be considered as linear coordinates $\xi_{1}, \xi_{2}, \xi_{3}, \xi_{4}, \xi_{5}$ on $\mathfrak{g}^{*}$, 
and the open stratum $\Omega_{1}$ is given by $\xi_{1} \neq 0$. The corresponding jump indices are $j_{1}=2, j_{2}=3, j_{3}=4$ and $j_{4}=5$. A cross-section for the orbits is given by $\Lambda_{1}$, the intersection of $\Omega_{1}$ with the hyperplane $\left\{\xi_{2}=\xi_{3}=\xi_{4}=\xi_{5}=0\right\}$. In this case $d=2$, and (2.3) becomes

$$
d \pi\left(X_{j}\right)= \begin{cases}i \xi_{1}, & j=1, \\ i t_{1}, & j=2, \\ i t_{2}, & j=3, \\ \xi_{1} \frac{\partial}{\partial t_{2}}, & j=4 \\ \frac{1}{2 \xi_{1}}\left(2 \xi_{1}^{2} \frac{\partial}{\partial t_{1}}+2 \xi_{1} t_{1} \frac{\partial}{\partial t_{2}}-i t_{2}^{2}\right), & j=5,\end{cases}
$$

all acting on $\mathcal{S}\left(\mathbb{R}^{2}\right)$.

Integrating, we see that

$$
\pi\left(\exp x_{j} X_{j}\right) f\left(t_{1}, t_{2}\right)= \begin{cases}e^{i x_{1} \xi_{1}} f\left(t_{1}, t_{2}\right), & j=1, \\ e^{i x_{2} t_{1}} f\left(t_{1}, t_{2}\right), & j=2, \\ e^{i x_{3} t_{2}} f\left(t_{1}, t_{2}\right), & j=3, \\ f\left(t_{1}, t_{2}+x_{4} \xi_{1}\right), & j=4, \\ e^{i(*)} f\left(t_{1}+x_{5} \xi_{1}, t_{2}+x_{5} t_{1}+\frac{1}{2} x_{5}^{2} \xi_{1}\right), & j=5,\end{cases}
$$

all acting on $L^{2}\left(\mathbb{R}^{2}\right)$. Here the factor in the exponential in the formula for

$$
\pi\left(\exp x_{5} X_{5}\right) f\left(t_{1}, t_{2}\right)
$$

is quite complicated:

$$
(*)=-\frac{x_{5}}{2 \xi_{1}} t_{2}^{2}-\frac{x_{5}^{2}}{2 \xi_{1}} t_{1} t_{2}-\frac{x_{5}^{3}}{6}\left(t_{2}+\frac{t_{1}^{2}}{\xi_{1}}\right)-\frac{x_{5}^{4}}{8} t_{1}-\frac{x_{5}^{5}}{40} .
$$

Now let $f_{1}, f_{2} \in \mathcal{S}\left(\mathbb{R}^{2}\right)$. The matrix coefficient $\left\langle\pi(g) f_{1}, f_{2}\right\rangle$, for fixed $g \in G$, as a function of $\xi_{1}$ (when $\left.\xi_{2}=\xi_{3}=\xi_{4}=\xi_{5}=0\right)$, is:

$$
\begin{aligned}
&\left\langle\pi\left(\exp x_{1} X_{1}\right)\right.\left.\pi\left(\exp x_{2} X_{2}\right) \pi\left(\exp x_{3} X_{3}\right) \pi\left(\exp x_{4} X_{4}\right) \pi\left(\exp x_{5} X_{5}\right) f_{1}, f_{2}\right\rangle \\
&= \int_{\mathbb{R}} \exp \left(i\left(x_{1} \xi_{1}+x_{2} t_{1}+x_{3} t_{2}+(*)\right)\right) \\
& \quad \cdot f_{1}\left(t_{1}+x_{5} \xi_{1}, t_{2}+x_{4} \xi_{1}+x_{5} t_{1}+\frac{1}{2} x_{5}^{2} \xi_{1}\right) \overline{f_{2}\left(t_{1}, t_{2}\right)} d t_{1} d t_{2}
\end{aligned}
$$

The exponential factor multiplied by the factor involving $f_{1}$ is clearly everywhere holomorphic in $\xi_{1}$ away from $\left\{\xi_{1}=0\right\}$, provided that $f_{1}$ has (ordinary) Fourier transform $\hat{f}_{1} \in C_{c}^{\infty}\left(\mathbb{R}^{2}\right)$ and thus $f_{1}$ lies in the Paley-Wiener class $\mathcal{P} \mathcal{W}\left(\mathbb{R}^{2}\right)$. Again, integration against the $f_{2}$ factor presents no difficulty provided $f_{2} \in C_{c}^{\infty}\left(\mathbb{R}^{2}\right)$. So for $f_{1} \in \mathcal{P} \mathcal{W}\left(\mathbb{R}^{2}\right), f_{2} \in C_{c}^{\infty}\left(\mathbb{R}^{2}\right)$, the matrix coefficient is holomorphic in $\left\{\xi_{1} \neq 0\right\}$.

Holomorphicity of matrix coefficients in the orbital parameters. We shall now deduce, as a consequence of Theorems 2.2 and 2.4, and a lemma which codifies the behavior exhibited by the two examples in the last subsection, the holomorphicity of the matrix coefficients of the irreducible unitary representations as a function of the orbital parameters. First, we show that in fact the matrix coefficients coming from the elements of the enveloping algebra are holomorphic. For the proof of the main result we only need that assertion for the elements of the Lie algebra, but we think the more general fact is interesting enough to warrant explicit mention. 
2.7. Proposition. For any element $E \in U(\mathfrak{g})$ (the universal enveloping algebra of $\mathfrak{g})$ and for every pair $f_{1}, f_{2} \in \mathcal{S}\left(\mathbb{R}^{d}\right)$, the function

$$
\left\langle d \pi_{\lambda}(E) f_{1}, f_{2}\right\rangle
$$

extends to a (rational) holomorphic function of $\lambda$ defined in the complexification $\left(\Lambda_{\varepsilon}\right)_{\mathbb{C}}$ of $\Lambda_{\varepsilon}$.

Proof. We apply Theorem 2.4 to the basis vectors $\left\{X_{j}\right\}$. Then it is clear that on the complexification $\left(\Lambda_{\varepsilon}\right)_{\mathbb{C}}$ of the variety $\Lambda_{\varepsilon}$, any infinitesimal matrix coefficient

$$
\left\langle d \pi_{\lambda}\left(X_{j}\right) f_{1}, f_{2}\right\rangle
$$

will be holomorphic (and rational) in $\lambda$. Since the matrix coefficient for any $X \in \mathfrak{g}$ will be a finite linear combination of these, the same is true of those matrix coefficients. Finally, from the form of the operators in (2.3), we see that the operators $d \pi_{\lambda}(E), E \in U(\mathfrak{g})$, involve only further products and derivatives of the polynomial functions $a_{X, r}$; and hence the matrix coefficients $\left\langle d \pi_{\lambda}(E) f_{1}, f_{2}\right\rangle$ are also rational with no poles in $\left(\Lambda_{\varepsilon}\right)_{\mathbb{C}}$.

In order to "integrate" the last result to obtain the main result, we need to compute the one-parameter unitary group of operators corresponding to the operator (2.3). This requires a further examination of Pedersen's coefficient functions $a_{X, r}^{\lambda}$, but this time we focus more carefully on their dependence on the parameter $t$.

2.8. Lemma. Fix a stratum $\Omega_{\varepsilon}$ and the corresponding affine cross-section $\Lambda_{\varepsilon}$. Let $2 d$ be the dimension of orbits in this stratum. Then there is an ordering of the variables $t_{1}, \ldots, t_{d}$ so that the coefficient functions $a_{X_{j}, r}^{\lambda}, 1 \leq r \leq d$, viewed as polynomials in $t=\left(t_{1}, \ldots, t_{d}\right) \in \mathbb{R}^{d}$, have the following property: For any $j, 1 \leq$ $j \leq m$, the operator $d \pi\left(X_{j}\right)$ is of the form

$$
d \pi\left(X_{j}\right) f(t)=i a_{X_{j}, 0}^{\lambda}(t) f(t)+\sum_{r=1}^{d} a_{X_{j}, r}^{\lambda}(t) \frac{\partial f}{\partial t_{r}}(t)
$$

where $a_{X_{j}, r}^{\lambda}(t)$ depends only on $t_{i}, i<r, 1 \leq r \leq d$. (In particular, the terms $\frac{\partial a_{X_{j}, r}^{\lambda}}{\partial t_{r}}(t) f(t)$ in (2.3) vanish.)

Proof. Pedersen obtains the formula (2.3) by showing that one can write

$$
\psi^{X_{j}}=a_{X_{j}, 0}^{\lambda}(q)+\sum_{r=1}^{d} a_{X_{j}, r}^{\lambda}(q) p_{r}
$$

where $\psi^{X_{j}}$ is the restriction to the orbit $\mathcal{O}$ corresponding to $\lambda$ of the linear functional $X_{j}$ on $\mathfrak{g}^{*}$, and where the $p$ 's and $q$ 's are canonical symplectic coordinates on $\mathcal{O}$ which he constructs. Now recall that since the $X_{j}$ 's are a Jordan-Hölder basis for the nilpotent Lie algebra $\mathfrak{g}$, we have $\left[X_{j}, X_{s}\right] \in \mathfrak{g}_{s-1}$ for any $j$ and $s$. In terms of symplectic geometry, this means that

$$
\left\{\psi^{X_{j}}, \psi^{X_{s}}\right\} \in \operatorname{span}\left(\psi^{X_{1}}, \ldots, \psi^{X_{s-1}}\right)
$$


where $\{$,$\} is the Poisson bracket on \mathcal{O}$. Now we need to carefully examine the arguments in $[\operatorname{Ped} 2, \S 5]$. Pedersen divides the jump indices of (2.1) into two disjoint ordered subsets, each containing $d$ elements: the "low" jump indices $\bar{l}_{1}<\cdots<\bar{l}_{d}$ and the "high" jump indices $k_{1}<\cdots<k_{d}$. For simplicity of notation we drop the bars on the $\bar{l}_{j}$ 's and also drop the implicit $\lambda$ 's everywhere. Then Pedersen shows that $\psi^{X_{j}}$ is a scalar for $j<l_{1}$, while $\psi^{X_{l_{1}}}=q_{1}$. Thus, using the facts that the $q$ 's Poisson-commute and that $\left\{p_{r}, q_{1}\right\}=\delta_{r 1}$, we have for any $j$ :

$$
\begin{aligned}
\left\{\psi^{X_{j}}, \psi^{X_{l_{1}}}\right\} & =\left\{a_{X_{j}, 0}(q)+\sum_{r=1}^{d} a_{X_{j}, r}(q) p_{r}, q_{1}\right\} \\
& =a_{X_{j}, 1}(q) \in \operatorname{span}\left(\psi^{X_{1}}, \ldots, \psi^{X_{l_{1}-1}}\right) \\
& =\text { scalar, }
\end{aligned}
$$

which is the case of the lemma for $r=1$. To get the other cases, observe from Pedersen's inductive construction of the $q_{r}$ 's that

$$
\psi^{X_{l_{r}}}=q_{r}+\operatorname{polynomial}\left(q_{1}, \ldots, q_{r-1}\right)
$$

and also that this marks the first occurrence of $q_{r}$. So one obtains similarly

$$
\begin{aligned}
\left\{\psi^{X_{j}}, \psi^{X_{l_{r}}}\right\} & =\left\{a_{X_{j}, 0}(q)+\sum_{s=1}^{d} a_{X_{j}, s}(q) p_{s}, q_{r}+\operatorname{polynomial}\left(q_{1}, \ldots, q_{r-1}\right)\right\} \\
& =a_{X_{j}, r}(q)+\operatorname{polynomial}\left(q_{1}, \ldots, q_{r-1}\right) \\
& =\operatorname{polynomial}\left(q_{1}, \ldots, q_{r-1}\right)
\end{aligned}
$$

which gives the general case of the lemma.

2.10. Remark. It is evident, for example from [Ped4], that Lemma 2.8 could not be true for a completely solvable group. It is the nilpotence that causes the latter terms in (2.3) to disappear. Now the simpler form (2.9) of the infinitesimal operators of the representation allows us to integrate them.

2.11. Proposition. Consider a differential operator $D$ on $\mathcal{S}\left(\mathbb{R}^{d}\right) \subseteq L^{2}\left(\mathbb{R}^{d}\right)$ of the form

$$
D f(t)=i p(t) f(t)+\sum_{r=1}^{d} q_{r}(t) \frac{\partial f}{\partial t_{r}}(t),
$$

where $p$ and the $q_{r}, 1 \leq r \leq d$, are real polynomial functions, and $q_{r}(t)$ depends only on $t_{j}, j<r$. Then for any $s \in \mathbb{R}$,

$$
e^{s D} f(t)=e^{i h(s, t)} f(t+k(s, t))
$$

where $h$ and $k$ are polynomials whose coefficients are explicit polynomials in the coefficients of $p$ and the $q_{r}, 1 \leq r \leq d$. The polynomial $k_{r}(s, t)$ satisfies $k_{r}(0, t)=0$, and only involves those $t_{j}$ 's with $j<r$.

Proof. Let $f \in \mathcal{S}\left(\mathbb{R}^{d}\right)$ and set $g(s, t)=e^{s D} f(t)$. Then $g$ satisfies the Cauchy problem

$$
\left\{\begin{array}{l}
g(0, t)=f(t) \\
\frac{\partial g}{\partial s}=D g(s, t)
\end{array}\right.
$$


Since the solution of this problem is unique, it is sufficient to verify that there is a solution of (2.12) of the form

$$
g(s, t)=e^{i h(s, t)} f(t+k(s, t))
$$

with $k_{r}(0, t)=0$ for all $r$, and $k_{r}(s, t)$ only involving those $t_{j}$ 's with $j<r$. Substituting the expression for $g$ in (2.12), we see that if there is such a solution, we need to take $h(0, t)=0$. Furthermore, equating coefficients of $e^{i h} f$ and of $e^{i h} \frac{\partial f}{\partial t_{r}}$ in (2.12) gives the partial differential equations

$$
\left\{\begin{array}{l}
\frac{\partial h}{\partial s}=p(t)+\sum_{r} q_{r}(t) \frac{\partial h}{\partial t_{r}}, \\
\frac{\partial k_{r}}{\partial s}=q_{r}(t)+\sum_{j<r} q_{j}(t) \frac{\partial k_{r}}{\partial t_{j}} .
\end{array}\right.
$$

These equations can be solved by a recursion technique. Write

$$
\left\{\begin{array}{l}
h(s, t)=s p(t)+s^{2} h_{2}(t)+s^{3} h_{3}(t)+\cdots, \\
k_{r}(s, t)=s q_{r}(t)+s^{2} k_{2}^{r}(t)+s^{3} k_{3}^{r}(t)+\cdots .
\end{array}\right.
$$

Substituting these expansions into the partial differential equations, one can solve uniquely for the $h_{j}$ 's and the $k_{j}^{r}$ 's. Furthermore, each power series in $s$ terminates after finitely many terms, so that one gets polynomials of the desired form. (This was the method for computing $(*)$ in Example 2.6.) It is also clear that the coefficients of $h$ and of $k_{r}$ are (real) polynomials in the coefficients of $p$ and the $q_{r}, 1 \leq r \leq d$.

For example, when $d=1$, the formula becomes

$$
\begin{aligned}
& \exp \left(s\left(i p(t)+q \frac{d}{d t}\right)\right) f(t) \\
& \quad=\exp \left(i\left(s p(t)+\frac{q s^{2}}{2} p^{\prime}(t)+\frac{q^{2} s^{3}}{3 !} p^{\prime \prime}(t)+\frac{q^{3} s^{4}}{4 !} p^{\prime \prime \prime}(t)+\cdots\right)\right) f(t+q s),
\end{aligned}
$$

which appeared in Example 2.5.

Now we are ready for the main result.

2.13. Theorem. Let $\Omega_{\varepsilon}$ be any stratum in Pedersen's stratification of $\mathfrak{g}^{*}$, and let $2 d$ be the dimension of the orbits in that stratum. The affine variety $\Lambda_{\varepsilon}$ parameterizes the representations corresponding to the G-orbits in $\Omega_{\varepsilon}$, all of which are realized in $L^{2}\left(\mathbb{R}^{d}\right)$. Then for any fixed $x \in G$, and for any pair $f_{1} \in \mathcal{P} \mathcal{W}\left(\mathbb{R}^{d}\right)$, $f_{2} \in C_{c}^{\infty}\left(\mathbb{R}^{d}\right)$, the matrix coefficient

$$
\left\langle\pi_{\lambda}(x) f_{1}, f_{2}\right\rangle
$$

extends to a holomorphic function of $\lambda \in\left(\Lambda_{\varepsilon}\right)_{\mathbb{C}}$.

Proof. First we show that for $f_{1} \in \mathcal{P} \mathcal{W}\left(\mathbb{R}^{d}\right)$, the function $\pi_{\lambda}(x) f_{1}(t)$ extends to be holomorphic in $t \in \mathbb{C}^{d}, x \in G_{\mathbb{C}}$, and $\lambda \in\left(\Lambda_{\varepsilon}\right)_{\mathbb{C}}$. To prove this, write

$$
\pi_{\lambda}(x) f_{1}(t)=\pi_{\lambda}\left(\exp x_{1} X_{1}\right) \pi_{\lambda}\left(\exp x_{2} X_{2}\right) \cdots \pi_{\lambda}\left(\exp x_{m} X_{m}\right) f_{1}(t),
$$


where $x=\left(x_{1}, \ldots, x_{m}\right) \in \mathbb{R}^{m}$ gives real analytic (in fact, polynomial) coordinates on $G$. We prove by descending induction on $j$ that

$$
\pi_{\lambda}\left(\exp x_{j} X_{j}\right) \cdots \pi_{\lambda}\left(\exp x_{m} X_{m}\right) f_{1}(t)
$$

extends to be holomorphic in $t \in \mathbb{C}^{d}, x_{j}, \ldots, x_{m} \in \mathbb{C}$, and $\lambda \in\left(\Lambda_{\varepsilon}\right)_{\mathbb{C}}$. To start the induction, $f_{1}$ is entire in $t$ since it is in the Paley-Wiener class. The inductive step follows immediately from Proposition 2.11. To get the conclusion of the theorem, observe that integration against $f_{2} \in C_{c}^{\infty}\left(\mathbb{R}^{d}\right)$ will (since $f_{2}$ has compact support) yield a smooth function of $x \in G$ and $\lambda \in\left(\Lambda_{\varepsilon}\right)_{\mathbb{C}}$, holomorphic in $\lambda$.

2.14. Remark. The proof of Theorem 2.13 reveals that the matrix coefficients of the operators arising from group elements are holomorphic, but unlike those arising from the enveloping algebra, they are not rational. As we saw in Example 2.5, it is also necessary to place further restrictions on $f_{1}, f_{2} \in \mathcal{S}\left(\mathbb{R}^{d}\right)$ in order for the corresponding matrix coefficient to be holomorphic.

2.15. Corollary. Let $\varphi$ be a distribution on $G$ with compact support. Then for any stratum $\Omega_{\varepsilon}$, the operator-valued function

$$
\pi_{\lambda}(\varphi)=\int_{G} \pi_{\lambda}(x) d \varphi(x)
$$

is weakly holomorphic on $\left(\Lambda_{\varepsilon}\right)_{\mathbb{C}}$, in the sense that $\left\langle\pi_{\lambda}(\varphi) f_{1}, f_{2}\right\rangle$ is holomorphic for any pair $f_{1} \in \mathcal{P} \mathcal{W}\left(\mathbb{R}^{d}\right), f_{2} \in C_{c}^{\infty}\left(\mathbb{R}^{d}\right)$.

Proof. This is almost immediate from Theorem 2.13. For any pair $f_{1} \in \mathcal{P} \mathcal{W}\left(\mathbb{R}^{d}\right)$, $f_{2} \in C_{c}^{\infty}\left(\mathbb{R}^{d}\right)$, the function

$$
\left\langle\pi_{\lambda}(\varphi) f_{1}, f_{2}\right\rangle=\int_{G}\left\langle\pi_{\lambda}(x) f_{1}, f_{2}\right\rangle d \varphi(x)
$$

is the pairing of $\left\langle\pi_{\lambda}(x) f_{1}, f_{2}\right\rangle$, which is a smooth function of $x$ and $\lambda$, holomorphic in $\lambda$ for fixed $x$, against a distribution of compact support in $x$. Hence the result is still a holomorphic function of $\lambda$.

Proof of Moss's conjecture. We conclude the section now with the proof of Moss's conjecture in the generality of an arbitrary simply connected nilpotent Lie group.

2.16. Theorem. Let $G$ be a simply connected nilpotent Lie group. Suppose that $\varphi$ is a distribution of compact support on $G$ with the property that $\hat{\varphi}_{\mathrm{op}}$ vanishes on a set of positive Plancherel measure in $\hat{G}$. Then $\varphi=0$.

Proof. We apply Corollary 2.15 to the top stratum $\Omega_{1}$. The result is that $\hat{\varphi}_{\mathrm{op}}$ is weakly holomorphic on $\left(\Lambda_{1}\right)_{\mathbb{C}}$. Now $\Omega_{1}$ has full Lebesgue measure in $\mathfrak{g}^{*}$, so $\Lambda_{1}$ corresponds to a set of full Plancherel measure in $\hat{G}$, and thus $\hat{\varphi}_{\mathrm{op}}$ is, by the hypothesis of the theorem, zero on a set of positive measure in $\Lambda_{1}$. That can only happen if $\varphi=0$. Indeed, any matrix coefficient of $\hat{\varphi}_{\mathrm{op}}$ with respect to a function in $\mathcal{P W}\left(\mathbb{R}^{d}\right)$ and a function in $C_{c}^{\infty}\left(\mathbb{R}^{d}\right)$ will be a holomorphic function on $\left(\Lambda_{1}\right)_{\mathbb{C}}$ and zero on a set of positive measure in $\Lambda_{1}$. But the variety $\left(\Lambda_{1}\right)_{\mathbb{C}}$ is connected (by the same argument as in the proof of Theorem 1.1), and therefore the matrix coefficient 
must vanish on $\Lambda_{1}$. Since the spaces $C_{c}^{\infty}\left(\mathbb{R}^{d}\right)$ and $\mathcal{P} \mathcal{W}\left(\mathbb{R}^{d}\right)$ are dense in $L^{2}\left(\mathbb{R}^{d}\right)$, the operator $\hat{\varphi}_{\text {op }}$ itself is zero on $\Lambda_{1}$. Now by an application of the Plancherel Theorem on $G$ we have that for any test function $\psi \in \mathcal{D}(G)$,

$$
\begin{aligned}
\langle\varphi, \psi\rangle & =\int_{\Lambda_{1}} \operatorname{Tr}\left[\hat{\varphi}_{\mathrm{op}}(\pi) \hat{\psi}_{\mathrm{op}}(\pi)^{*}\right] d \mu(\pi) \\
& =0 \quad \forall \psi
\end{aligned}
$$

That is, $\varphi=0$.

\section{VAnishing of the Dixmier-Douady Class FOR CONTINUOUS-TRACE SUBQUOTIENTS OF THE GROUP $C^{*}$-ALGEBRA OF A NILPOTENT LIE GROUP}

Statement of the problem and the conjecture. In the introduction to this paper, we already indicated that the operator-valued Fourier transform of a function $\varphi \in C_{c}^{\infty}$ may be viewed as an element of the group $C^{*}$-algebra $C^{*}(G)$. In general this is a very complicated object; for a nilpotent Lie group, about the most one knows about $C^{*}(G)$ is that it is a (generalized continuous-trace) liminary $C^{*}$-algebra with a finite composition series into continuous-trace subquotients [Dix1]. Recall here that continuous-trace $C^{*}$-algebras have Hausdorff spectrum and are the basic building blocks for all type I $C^{*}$-algebras [Fell]. By the basic theory of DixmierDouady, a stable continuous-trace $C^{*}$-algebra $A$, or in fact any $\aleph_{0}$-homogeneous continuous-trace $C^{*}$-algebra $A$ with finite-dimensional spectrum, is the algebra of sections of a locally trivial bundle of algebras over the locally compact Hausdorff space $\hat{A}$, with fibers isomorphic to the compact operators on an infinite-dimensional separable Hilbert space. Such a bundle is trivial if and only if its Dixmier-Douady invariant, which lies in $H^{3}(\hat{A}, \mathbb{Z})$, vanishes. So to understand the operator-valued Fourier transform on a nilpotent Lie group, we necessarily encounter:

3.1. Question. Can $C^{*}(G)$ have a continuous-trace subquotient with non-zero Dixmier-Douady invariant?

If the answer is yes, then the non-trivial bundle structure of this subquotient is an essential feature in understanding the behavior of operator-valued Fourier transforms. If the answer is no, then it makes sense to understand the operatorvalued Fourier transform (or at least its image in any continuous-trace subquotient) as an operator-valued function on $\hat{G}$. In [RaRo, $\S 4]$ and in [Ros, $\S 3]$, we raised this question and conjectured the following:

3.2. Conjecture. If $G$ is a connected nilpotent Lie group, every continuous-trace subquotient of $C^{*}(G)$ has vanishing Dixmier-Douady invariant.

We showed in [RaRo, $\S 4]$ and in [Ros, $\S 3]$ this is not the case for connected type I solvable Lie groups, nor is it the case for disconnected Lie groups in which the connected component of the identity is abelian and has finite index in $G$, but we gave some evidence for the truth of the corresponding conjecture for linear semisimple Lie and $\mathfrak{p}$-adic groups.

Proof of the conjecture for two-step nilpotent Lie groups. Using an analysis related to our study in $\S 1$ of the Fourier transform for nilpotent Lie groups with 
"generically flat" orbits, we will now prove Conjecture 3.2 for two-step nilpotent Lie groups. The argument is interesting in that semi-simple Lie groups play an essential role in the proof.

3.3. Lemma. Let $G$ be any two-step nilpotent Lie group with Lie algebra $\mathfrak{g}$. If $A$ is a subquotient of $C^{*}(G)$ with Hausdorff spectrum, then there is no convergent sequence $\pi_{n} \rightarrow \pi$ in $\hat{A}$ such that each $\pi_{n}$ corresponds to an orbit $\mathcal{O}_{n}$ in $\mathfrak{g}^{*}$ of dimension $2 r$ but such that $\pi$ corresponds to an orbit $\mathcal{O}$ in $\mathfrak{g}^{*}$ of dimension different from $2 r$.

Proof. Recall that $\hat{G}$ is canonically homeomorphic to the "integral" part of $\mathfrak{g}^{*} / G$. ("Integrality" is a vacuous condition if $G$ is simply connected.) Now the orbits $\mathcal{O}_{n}$ cannot converge to an orbit of bigger dimension (since the subset of $\mathfrak{g}^{*}$ consisting of orbits of dimension $\leq 2 r$ is Zariski-closed (cf. [Dix2, Proposition 1.11.5]) and thus closed in the Hausdorff topology), so we have to rule out the possibility that $\mathcal{O}$ could have strictly smaller dimension than $2 r$. However, if this is the case and $\pi_{n} \rightarrow \pi$ in $\hat{G}$, then in fact the sequence $\left\{\pi_{n}\right\}$ must have a whole affine space of limit points in $\hat{G}$. (This is clear since all the $G$-orbits are flat; cf. Proposition 1.6.) Since $\hat{A}$ is Hausdorff and is a locally closed subset of $\hat{G}$, we get a contradiction.

3.4. Theorem. Let $G$ be any connected two-step nilpotent Lie group with Lie algebra $\mathfrak{g}$. If $A$ is a subquotient of $C^{*}(G)$ with continuous trace, then $A$ has vanishing Dixmier-Douady invariant (in other words, is Morita-equivalent to a commutative $C^{*}$-algebra).

Proof. By Lemma 3.2, each connected component of $\hat{A}$ consists only of representations corresponding to coadjoint orbits of some fixed dimension. So we may as well assume $\hat{A}$ consists only of representations corresponding to coadjoint orbits of some fixed dimension $2 r$. (In fact in the two-step case, the orbits of fixed dimension $2 r$ do indeed give a continuous-trace subquotient of $C^{*}(G)$, as pointed out in Corollary 6.3.4 of [Echt].)

Since the $C^{*}$-algebra of any two-step nilpotent Lie group is a quotient of the $C^{*}$-algebra of the universal two-step nilpotent Lie group $G_{n}$ on some number $n$ of generators (with Lie algebra $\mathfrak{g}_{n}$ having a basis $x_{1}, \ldots, x_{n}, z_{i j}=\left[x_{i}, x_{j}\right], 1 \leq i<j \leq$ $n$, where the $z_{i j}$ 's span the center $\mathfrak{z}_{n}$ ), it is enough to consider this case. Note that for any $f \in \mathfrak{g}_{n}^{*}$, the alternating bilinear form $B_{f}$ only depends on the restriction of $f$ to $\mathfrak{z}_{n}$, and descends to an alternating form $\omega_{f}$ on $\mathfrak{g}_{n} / \mathfrak{z}_{n} \cong \mathbb{R}^{n}$. Also, knowledge of $\omega_{f}$ is equivalent to knowledge of $\left.f\right|_{\mathfrak{z}_{n}}$, since the matrix entries of $\omega_{f}$ are

$$
\left(\omega_{f}\right)_{i j}=B_{f}\left(x_{i}, x_{j}\right)=f\left(\left[x_{i}, x_{j}\right]\right)= \begin{cases}f\left(z_{i j}\right), & i<j \\ 0, & i=j \\ -f\left(z_{j i}\right), & i>j\end{cases}
$$

and the $z_{i j}$ are a basis for $\mathfrak{z}_{n}$. The orbit $G_{n} \cdot f$ is $f+\left(\operatorname{rad} B_{f}\right)^{\perp}$, whereas the set of functionals giving rise to the form $\omega_{f}$ is $f+\mathfrak{z}_{n}^{\perp}$. Thus $\omega_{f}$ only depends on the $G$-orbit of $f$, and the map $G \cdot f \mapsto \omega_{f}$ identifies the subspace $\Lambda_{n, r}$ of $\mathfrak{g}_{n}^{*} / G_{n}$ consisting of orbits of dimension $2 r$ with the total space of a vector bundle of rank $n-2 r$ over the space $X_{n, r}$ of skew-symmetric bilinear forms on $\mathbb{R}^{n}$ of rank $2 r$, $1 \leq r \leq\left[\frac{n}{2}\right]$. Since any such form is conjugate under the action of $G L(n, \mathbb{R})$ to the 
standard form given by

$$
\left(\begin{array}{ccc}
0 & I_{r} & 0 \\
-I_{r} & 0 & 0 \\
0 & 0 & 0
\end{array}\right)
$$

one finds that

$X_{n, r} \cong G L(n, \mathbb{R}) /\left\{\left(\begin{array}{cc}A & B \\ 0 & D\end{array}\right): A \in S p(2 r, \mathbb{R}), D \in G L(n-2 r, \mathbb{R}), B\right.$ arbitrary $\}$,

which (if $\simeq$ denotes "is homotopy-equivalent to") gives

$$
\Lambda_{n, r} \simeq X_{n, r} \simeq O(n) / U(r) \times O(n-2 r) \simeq S O(n) / U(r) \times S O(n-2 r) .
$$

This is a quotient of a connected compact Lie group by a connected subgroup of maximal rank, and thus is diffeomorphic to the quotient of a complex semisimple group by a parabolic subgroup, in other words, a generalized flag variety. It thus (by Bruhat decomposition) has a cell decomposition with only even-dimensional cells, and in particular its odd-dimensional cohomology vanishes. So $H^{3}\left(X_{n, r}, \mathbb{Z}\right)=0$ and the Dixmier-Douady invariant vanishes.

The analysis in $\S 1$ of nilpotent Lie groups $G$ having generic representations square-integrable modulo their projective kernels suggests that one can do a similar analysis to prove vanishing of the Dixmier-Douady invariant for the "large" continuous-trace ideal in $C^{*}(G)$.

\section{REFERENCES}

[Ando] S. Ando, Paley-Wiener type theorem for Heisenberg groups, Proc. Japan Acad. 52 (1976), 331-333. MR 55:5796

[ArnG] D. Arnal and S. Gutt, Décomposition de $L^{2}(G)$ et transformation de Fourier adaptée pour un groupe $G$ nilpotent, C. R. Acad. Sci. Paris Sér. I Math. 306 (1988), 25-28. MR 88m:22015

[Br] I. Brown, Dual topology of a nilpotent Lie group, Ann. Sci. École Norm. Sup. 6 (1973), 407-411. MR 50:4813

[CorGr] L. Corwin and F. P. Greenleaf, Representations of nilpotent Lie groups and their Applications, Part 1: Basic Theory and Examples, Cambridge Studies in Advanced Math., vol. 18, Cambridge Univ. Press, Cambridge, New York, 1990. MR 92b:22007

[Dix1] J. Dixmier, Sur le dual d'un groupe de Lie nilpotent, Bull. Sci. Math. 90 (1966), 113118. MR 35:298

[Dix2] J. Dixmier, Enveloping Algebras, North-Holland Math. Library, vol. 14, North-Holland, Amsterdam, New York, Oxford, 1977. MR 58:16803b

[Echt] S. Echterhoff, Crossed products with continuous trace, Habilitationsschrift, Universität-Gesamthochschule Paderborn, Paderborn, 1993.

[Fell] J. M. G. Fell, The structure of algebras of operator fields, Acta Math. 106 (1961) 233-280. MR 29:1547

[Hel] S. Helgason, The Radon Transform, Progress in Math., vol. 5, Birkhäuser, Boston, Basel, Stuttgart, 1980. MR 83f:43012

[Joy] K. T. Joy, A description of the topology on the dual space of a nilpotent Lie group, Pacific J. Math. 112 (1984), 135-139. MR 85e:22013

[Kum] K. Kumahara, An analogue of the Paley-Wiener Theorem for the Heisenberg group, Proc. Japan Acad. 47 (1971), 491-494. MR 46:9244

[MoorWo] C. C. Moore and J. A. Wolf, Square integrable representations of nilpotent groups, Trans. Amer. Math. Soc. 185 (1973), 445-462. MR 49:3033 
[Moss] J. D. Moss, Jr., A Paley-Wiener Theorem for selected nilpotent Lie groups, J. Funct. Anal. 114 (1993), 395-411. MR 56:11992

[Mum] D. Mumford, Algebraic Geometry, I: Complex Projective Varieties, Grundlehren der math. Wiss., vol. 221, Springer-Verlag, Berlin, Heidelberg, New York, 1976. MR 94e:22017

[Park] R. Park, A Paley-Wiener Theorem for all two- and three-step nilpotent Lie groups, Preprint (1994).

[Ped1] N. V. Pedersen, On the symplectic structure of coadjoint orbits of (solvable) Lie groups and applications, I, Math. Ann. 281 (1988), 633-669. MR 90c:22024

[Ped2] N. V. Pedersen, Geometric quantization and the universal enveloping algebra of a nilpotent Lie group, Trans. Amer. Math. Soc. 315 (1989), 511-563. MR 90c:22026

[Ped3] N. V. Pedersen, Geometric quantization and nilpotent Lie groups: a collection of examples, Univ. of Copenhagen, Copenhagen, 1988.

[Ped4] N. V. Pedersen, Orbits and primitive ideals of solvable Lie algebras, Math. Ann. 298 (1994), 275-326. MR 94m:17009

[Puk] L. Pukanszky, On Kirillov's character formula, J. reine ang. Math. 311 (1979), 408440. MR 81b:22014

[RaRo] I. Raeburn and J. Rosenberg, Crossed products of continuous-trace $C^{*}$-algebras by smooth actions, Trans. Amer. Math. Soc. 305 (1988), 1-45. MR 89e:46077

[Ros] J. Rosenberg, $C^{*}$-algebras and Mackey's theory of group representations, $C^{*}$-algebras: 1943-1993, a Fifty Year Celebration (R. S. Doran, ed.), Contemp. Math., vol. 167, Amer. Math. Soc., Providence, RI, 1994, pp. 151-181. MR 94:17

[ScSi] D. Scott and A. Sitaram, Some remarks on the Pompieu problem for groups, Proc. Amer. Math. Soc. 104 (1988), 1261-1266. MR 89g:43009

[Sev1] L. A. Sevastyanov, The Paley-Wiener Theorem for the Heisenberg group, Trudy Univ. Druzby Narod. 80 (1976), no. 9, 122-126. MR 58:28277

[Sev2] L. A. Sevastyanov, An analogue of the Paley-Wiener Theorem for a nilpotent Lie group, An analogue of the Paley-Wiener Theorem for metabelian Lie groups, Topological Spaces and Their Mappings, Latv. Gos. Univ. Riga, Riga, 1981, pp. 130-136, 178, 184-185. MR 84a:22024

[Shaf] I. Shafarevich, Basic Algebraic Geometry, transl. by K. A. Hirsch, Grundlehren der math. Wiss., vol. 213, Springer-Verlag, Berlin, Heidelberg, New York, 1974 and 1977. MR 51:3163

Department of Mathematics, University of Maryland, College Park, Maryland 20742

E-mail address: rll@math.umd.edu; jmr@math.umd.edu 